\title{
Transcription factor TabHLH49 positively regulates dehydrin WZY2 gene expression and enhances drought stress tolerance in wheat
}

\author{
Hao Liu ${ }^{1 \dagger}$, Ying Yang ${ }^{1,2+}$, Dandan Liu ${ }^{3 \dagger}$, Xiaoyu Wang ${ }^{1,4}$ and Linsheng Zhang ${ }^{1 *}$
}

\begin{abstract}
Background: As functional proteins, dehydrins are found in many maturing seeds and vegetable tissues under adverse environmental conditions. However, the regulation of dehydrin expression remains unclear.

Results: In this study, a novel drought stress-related bHLH transcription factor, TabHLH49, was isolated from a wheat cDNA library treated with the drought and cold stress by using yeast one-hybrid system. TabHLH49 protein possesses a typical conserved bHLH domain and is a Myc-type bHLH transcription factor. TabHLH49 was detected in the nucleus of tobacco epidermal cells, and the amino acid sequences at the C-terminus (amino acids 323-362) is necessary for its transactivation activity. Real-time PCR analyses revealed the tissue-specific expression and drought stress-responsive expression of TabHLH49 in wheat. In addition, the verification in $\mathrm{Y} 1 \mathrm{H}$ and electrophoretic mobility shift assays illustrated that TabHLH49 protein can bind and interact with the promoter of the wheat WZY2 dehydrin. Furthermore, the dual-luciferase assays showed that TabHLH49 can positively regulate the expression of WZY2 dehydrin. The transient expression and BSMV-mediated gene silencing of TabHLH49 also showed that TabHLH49 positively regulates the expression of WZY2 dehydrin and improves drought stress resistance in wheat.

Conclusions: These results provide direct evidences that TabHLH49 positively regulates expression level of dehydrin WZY2 gene and improves drought tolerance of wheat.
\end{abstract}

Keywords: Wheat, Dehydrin, Drought stress, bHLH transcription factor, Regulation mechanism

\section{Background}

As an important food crop, wheat has been widely cultivated in most parts of northern China. However, the plants typically suffer from the different types of abiotic stresses including drought, cold damage, or salt injury during their growth and development periods, which can directly affect the overall wheat production.

\footnotetext{
* Correspondence: linszhang@nwsuaf.edu.cn

${ }^{\dagger}$ Hao Liu, Ying Yang and Dandan Liu contributed equally to this work.

${ }^{1}$ College of Life Science/State Key Laboratory of Crop Stress Biology for Arid Areas, Northwest A\&F University, Yangling 712100, China

Full list of author information is available at the end of the article
}

As sessile organisms, plants have evolved the ability to synthesize a series of stress-responsive proteins to avoid or defend against adverse conditions. Late embryogenesis abundant (LEA) proteins act as functional proteins in helping to protect cells from direct dehydration. Based on their conserved sequence motifs, the LEA proteins have been grouped into seven distinctive groups [1]. Group 2 LEA proteins, also known as dehydrins, are characterized by their highly hydrophilicity. They are considered hydrophiles and contain three typical conserved motifs: K-(EKKGIMDKIKEKLPG), Y-([V/T]D [E/ Q]YGNP) and S-(serine-track) segments. Based on the

C C The Author(s). 2020 Open Access This article is licensed under a Creative Commons Attribution 4.0 International License, which permits use, sharing, adaptation, distribution and reproduction in any medium or format, as long as you give appropriate credit to the original author(s) and the source, provide a link to the Creative Commons licence, and indicate if changes were made. The images or other third party material in this article are included in the article's Creative Commons licence, unless indicated otherwise in a credit line to the material. If material is not included in the article's Creative Commons licence and your intended use is not permitted by statutory regulation or exceeds the permitted use, you will need to obtain permission directly from the copyright holder. To view a copy of this licence, visit http://creativecommons.org/licenses/by/4.0/ The Creative Commons Public Domain Dedication waiver (http://creativecommons.org/publicdomain/zero/1.0/) applies to the data made available in this article, unless otherwise stated in a credit line to the data. 
presence of these conserved sequences (K-, Y-, and Ssegments), dehydrins are classified into the different subclasses: YnKn, YnSKn, KnS, SKn and Kn [2]. The wheat dehydrin WZY2 investigated in the current study is a YSK2-type dehydrin belonging to the YnSKn subtype [3].

The basic helix-loop-helix (bHLH) proteins are the second largest superfamily of plant transcription factors and can play important roles in hormone signaling, photomorphogenesis, and secondary metabolism. This family is well known due to its conserved basic-helixloop-helix structural domain consisting of two functional regions, the basic amino acid region and the helix-loophelix region $(\mathrm{HLH})$ located at the $\mathrm{N}$-terminus and $\mathrm{C}$ terminus of the bHLH domain, respectively. The basic amino acid region contains basic residues that can recognize and specifically bind the DNA motif in target genes [4]. The helix-loop-helix region consists of two amphipathic alpha helices linked by a loop with variable length and amino-acid composition, which can promote the formation of homodimers or heterodimers to control gene transcription [5].

According to previous studies on the bHLH proteins, these plant transcription factors are widely involved in the regulation of plant physiological and metabolic pathways. For example, bHLH factors play an important biological role in the regulatory network controlling anther development in Arabidopsis thaliana [6, 7]. In addition, bHLHs are also involved in plant responses to drought, salinity, and chilling abiotic stress. AtbHLH112, a nuclear-localized protein induced by salt, drought and abscisic acid (ABA), encodes a bHLH protein. AtbHLH112 serves as a transcriptional activator that regulates the expression of genes via binding to their GCG- or E-boxes to mediate physiological responses, including proline biosynthesis and ROS scavenging pathways, to enhance stress tolerance [8]. Moreover, a bHLH gene ThbHLH1 from Tamarix hispida can improve abiotic stress tolerance by enhancing osmotic potential and decreasing reactive oxygen accumulation [9].

Many previous studies, including our studies, have shown that wheat synthesizes dehydrins when under abiotic stress. However, the upstream regulatory mechanism and function of dehydrins thus far remains unclear. In this study, we have further explored the stress response mechanism of the WZY2 (UniProt ID: BOLXL4) dehydrin based on our cloned promoter of WZY2 from wheat "ZhengYin 1\#" in water stress. TabHLH49 (UniProt ID: A0A3B6RCI9) was isolated and identified in the wheat cDNA library treated with drought and cold stress by using the yeast one-hybrid system. TabHLH49 protein encodes a basic helix-loop-helix (bHLH) transcription factor that functions as a positive regulator of WZY2 dehydrin gene expression. This is the first detailed report of a bHLH involved in the mechanism of dehydrin regulation under abiotic stress and clarifies its role in the WZY2 dehydrin signaling pathway.

\section{Results}

Characterization of TabHLH49 and bioinformatics analysis In our previous study, we isolated a cDNA segment of bHLH49-like protein interacting with dehydrin WZY2 promoter performed by yeast one-hybrid $(\mathrm{Y} 1 \mathrm{H})$ assay [10], the complete coding sequence was obtained via a BLAST search against the wheat genomic database, and this gene was named TabHLH49. TabHLH49 encoded 440 amino acids with a predicted protein molecular weight of $47.54 \mathrm{kDa}$ and an isoelectric point of 5.61 (Fig. 1a). This gene contained a conserved bHLH domain and belonged to the bHLH transcription factor family. The three-dimensional structure of TabHLH49 was constructed by SWISS-MODLE software (Fig. 1c). The phylogenetic tree was constructed using TabHLH49 and $162 \mathrm{bHLH}$ gene sequences from Arabidopsis thaliana (Fig. 1b). The results indicated that TabHLH49 was highly homologous to AtbHLH049 and was a Myc-type bHLH transcription factor.

\section{Nuclear localization and transactivation activity of TabHLH49}

According to the ProtComp prediction algorithm, the nuclear localization signal 'VSCPKKRKRPSQ' was present in the amino acid sequence of TabHLH49 (Fig. 1a). Therefore, TabHLH49 may be a nuclear localization protein. To test this reasoning, the subcellular localization of TabHLH49 was observed by coexpression of TabHLH49-GFP under the control of the 35S promoter. The recombinant vector was transformed into Agrobacterium tumefaciens strain GV3101 and then infiltrated into tobacco leaves. GV3101 cells transformed with the 35S:GFP vector were infiltrated into tobacco leaves as control. The results indicated that the green fluorescence of TabHLH49: GFP fusion protein was only in the nucleus of the tobacco epidermal cells, while the empty control was evenly distributed throughout the tobacco cells (Fig. 2a). This finding indicated that TabHLH49 is specifically localized to the nucleus.

The transactivation activity of the TabHLH49 protein was determined in $\mathrm{Y} 2 \mathrm{H}$ Gold yeast cells. The expression of the reporter gene could not be activated when the Cterminus (amino acids 323-362) was absent, indicating that the amino acid sequences at the $\mathrm{C}$-terminus (amino acids 323-362) are necessary for the transactivation activity of TabHLH49 (Fig. 2c).

\section{Expression patterns analysis of TabHLH49 and WZY2 in wheat}

Real-time PCR was used to detect the expression of TabHLH49 in different tissues of wheat (Fig. 3a). The 


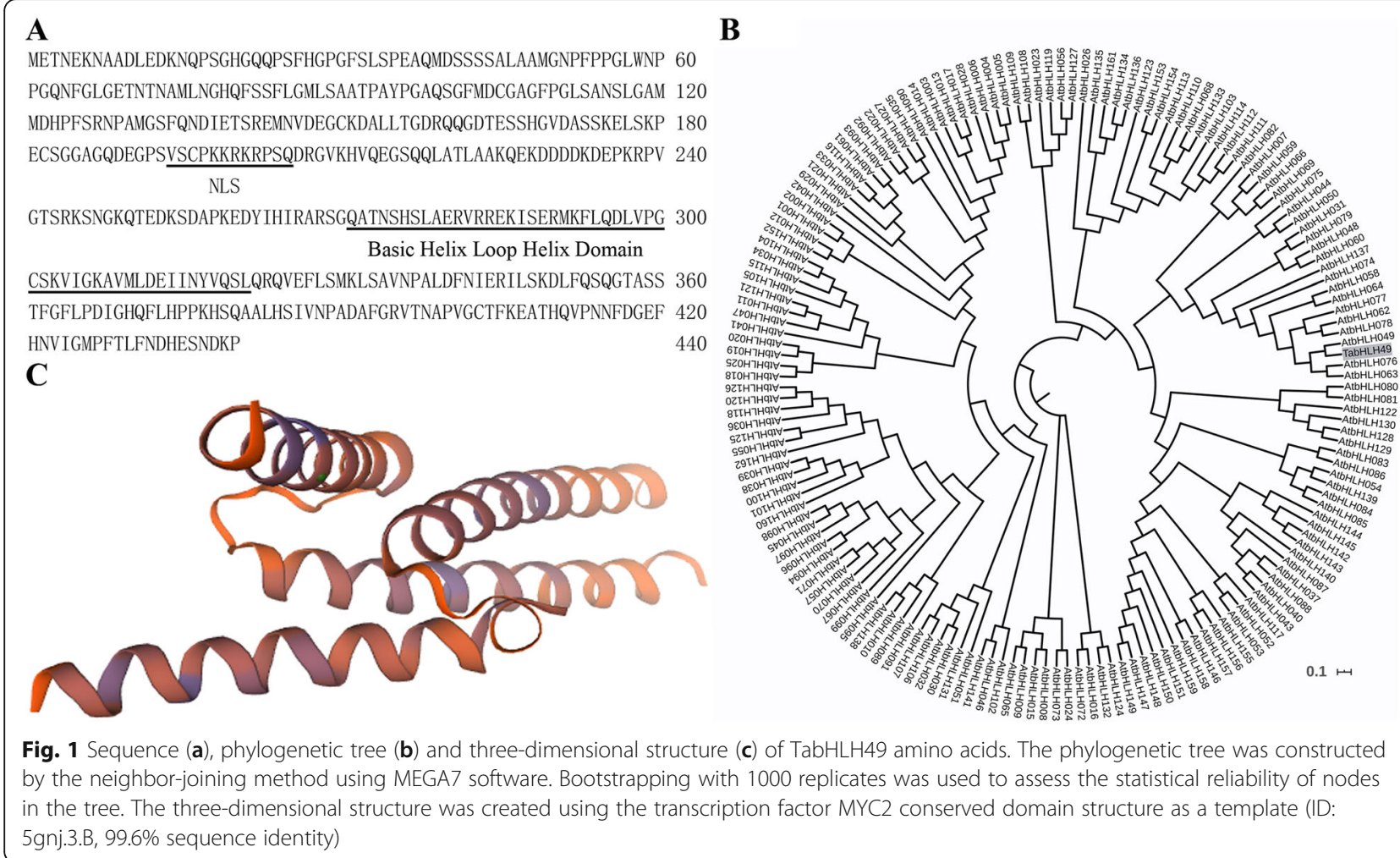

results showed that TabHLH49 was expressed in the roots, stems and leaves of 2-week-old seedlings. The expression of TabHLH49 was abundantly accumulated in roots (1.71-fold) and leaves (2.89-fold) compared to stems.

Our previous studies showed that the expression of WZY2 was highest in leaves during the wheat seedling stage [11]. Therefore, we selected the leaves of wheat seedlings to study the expression levels of TabHLH49 and WZY2 at different time points $(0,1,4,8$ and $12 \mathrm{~h})$ after PEG treatment (Fig. 3b). Real-time PCR results showed that the relative expression level of TabHLH49 obviously increased over $1-12 \mathrm{~h}$ and reached the highest point, approximately a 31.6 -fold change, at $12 \mathrm{~h}$ compared to $0 \mathrm{~h}$. Interestingly, the expression of $W Z Y 2$ showed a similar tendency as TabHLH49 but lagged behind TabHLH49. These results indicated that TabHLH49 may positively regulate the expression of WZY2.

\section{TabHLH49 is an upstream regulator of WZY2}

To examine the interaction between TabHLH49 and the WZY2 promoter (Pwzy2), we performed yeast onehybrid and EMSA assays. The yeast one-hybrid results showed that $\mathrm{Y} 1 \mathrm{H}$ Gold yeast cells containing pGADT7TabHLH49 could not grow on SD/-Leu/AbA ${ }^{200}$ selective medium. However, Y1H Gold (Pwzy2) bait yeast strains containing pGADT7-TabHLH49 were able to grow on $\mathrm{SD} /$-Leu and $\mathrm{SD} /-\mathrm{Leu} / \mathrm{AbA}^{200}$ selective medium (Fig. 4a and Fig. S1). The EMSA experiments showed that the gel mobility of Pwzy2 changed after incubation with TabHLH49 protein (Fig. 4b and Fig. S2). These results indicated that TabHLH49 is an upstream regulator of $W Z Y 2$.

TabHLH49 positively regulates the expression of WZY2 and improves the drought tolerance in wheat

To characterize the ability of TabHLH49 to activate the $W Z Y 2$ gene, tobacco leaves were co-infiltrated with $A$. tumefaciens containing the 35S:TabHLH49:GFP vector together with a Renilla luciferase gene driven by the WZY2 promoter. The Rluc/Fluc ratio was significantly increased by simultaneous TabHLH49:GFP overexpression compared to GFP expression (Fig. 5b).

To explore the function of TabHLH49 in wheat, $A$. tumefaciens GV3101 with 35S:TabHLH49:GFP (OE) and 35S:GFP (CK) were injected into wheat leaves for transient gene expression assays, as previously described [12, 13]. After natural drought for $10 \mathrm{~d}$, the wheat leaves overexpressing GFP showed obvious wilting and curling; however, wheat that overexpressed TabHLH49:GFP showed better growth (Fig. 5c). Real-time PCR results showed that the expression of TabHLH49 and WZY2 was significantly increased in wheat leaves overexpressing TabHLH49:GFP compared to control after drought stress (Fig. 5d-e). We also determined the content of 

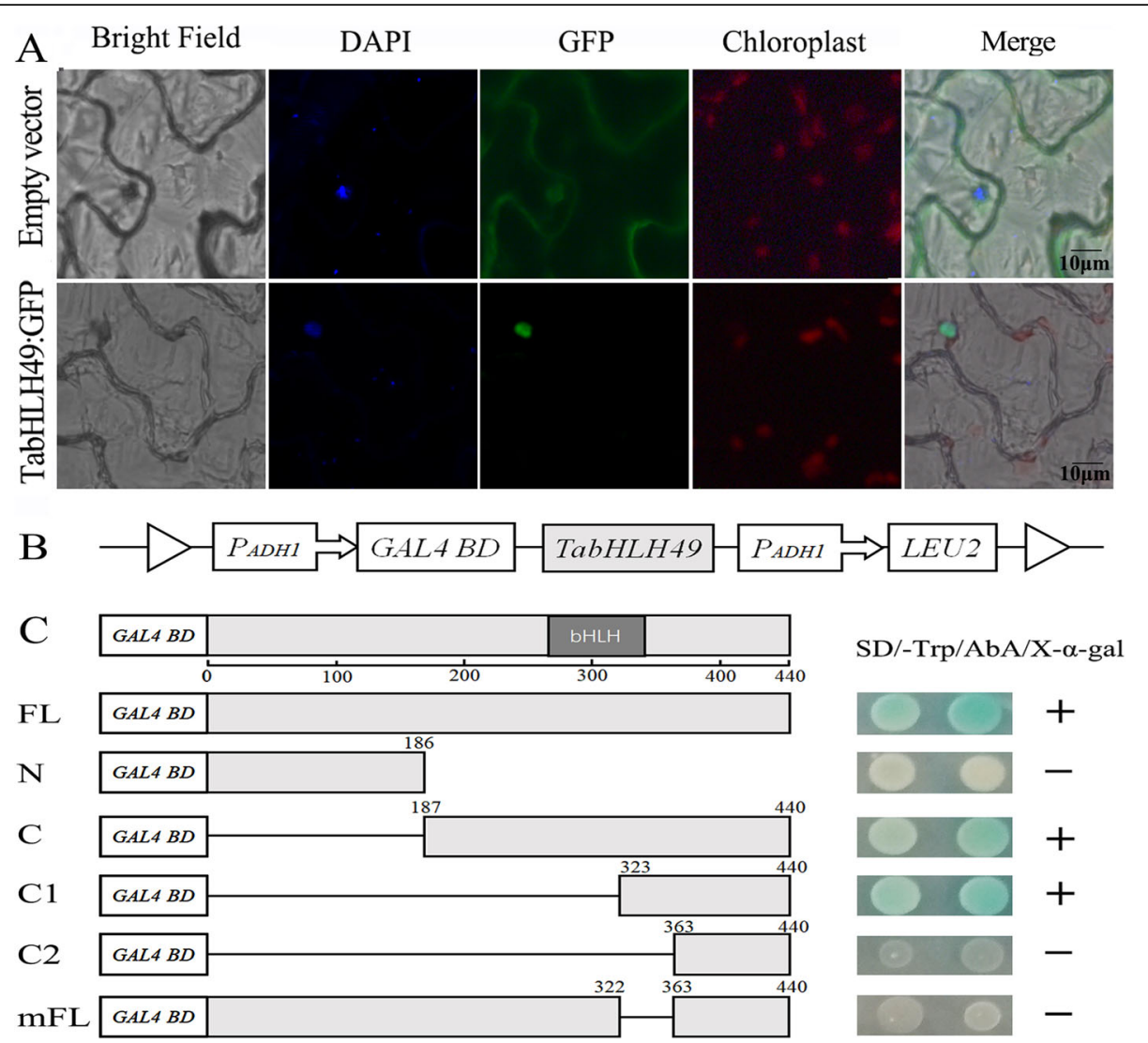

Fig. 2 Subcellular localization (a) and transactivation assay (b and c) of TabHLH49 protein. a Subcellular localization of TabHLH49 in tobacco epidermal cells. GFP and TabHLH49:GFP under the control of the CaMV35S promoter were separately transiently expressed in tobacco epidermal cells. Scale bar $=10 \mu \mathrm{m}$. b Diagram of the pGBKT7 construct that expressed different truncated TabHLH49 proteins in yeast. c Transactivation activities of the intact or truncated TabHLH49. Fusion proteins of the GAL4 DNA-binding domain and different portions of TabHLH49 were transformed into $\mathrm{Y} 2 \mathrm{H}$ Gold cells and grown on SD/-Trp/X-a-gal/AbA plates to assess their transactivation activities

malondialdehyde (MDA), relative water content and chlorophyll content in the CK and OE lines (Fig. 5f-h). After drought stress, OE lines showed significantly lower MDA content, higher relative water content and higher chlorophyll content than CK lines.
We also explored the function of the TabHLH49 gene in wheat using the barley stripe mosaic virus-induced gene silencing (BSMV-VIGS) technique. The BSMV construct, BSMV-TabHLH49, carrying a $459 \mathrm{bp}$ fragment from the TabHLH49 cDNA sequence, was used to
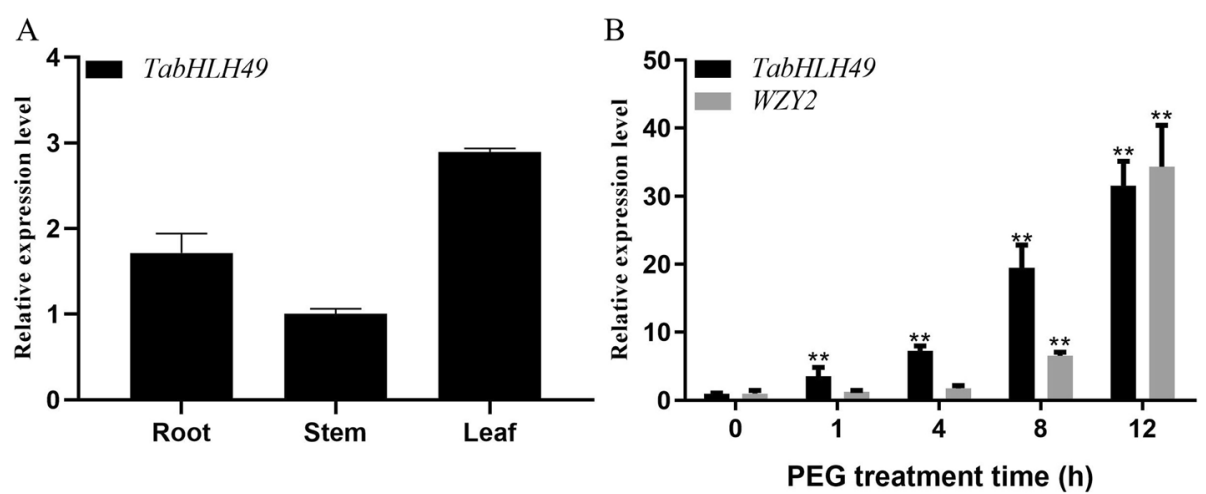

Fig. 3 Expression patterns of TabHLH49 and WZY2 genes determined by real-time PCR. a Real-time PCR was used to analyze the expression levels of TabHLH49 in different tissues. b Expression of TabHLH49 and WZY2 genes in response to 20\% PEG 6000 treatment. The values are the mean \pm SD from three samples, and significant differences are indicated as $p<0.05\left(^{*}\right)$ and $p<0.01\left(^{* *}\right)$ 

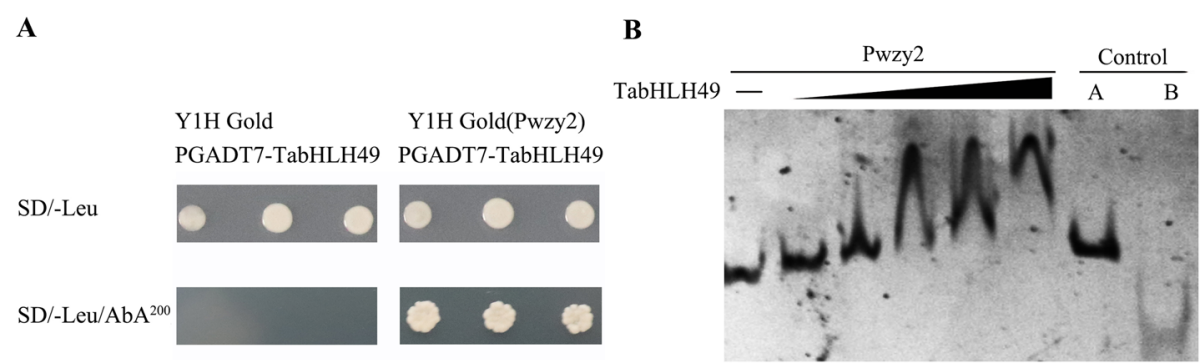

Fig. 4 TabHLH49 interacts with the promoter of WZY2 (Pwzy2). a Interaction of TabHLH49 with the promoter of WZY2 in the Y1H assay. $\mathbf{b}$ EMSA was performed to analyze the interactions between His6-TabHLH49 and the WZY2 promoter. Increasing amounts of TabHLH49 (0.1, $0.2,0.4,0.6$ and $0.8 \mu \mathrm{M}$ ) were used. As negative controls, bovine serum albumin (BSA) instead of His6-TabHLH49 (control A) and (GAGCGTAACTGCCCACCA CTCACTGGCTCACGCGCTGCCC) repeated tandem DNA fragment instead of the WZY2 promoter (control B) were included in the binding assays

silence TabHLH49 gene in wheat seed (Table S1). Wheat leaves photobleaching was observed in all plants infected with the BSMV-PDS construct at 10 days post inoculation (dpi), suggesting that the endogenous PDS gene was silenced (Fig. 6a). After natural drought for $10 \mathrm{~d}$, the BSMV-TabHLH49 lines withered and curled more severely than the BSMV-0 and BSMV-PDS lines (Fig. 6a). Real-time PCR analysis showed that the transcript levels of TabHLH49 and WZY2 were reduced in the BSMVTabHLH49 lines compared with the BSMV:0 and BSMV-PDS lines after drought stress (Fig. 6b-c). BSMVTabHLH49 lines showed obviously higher MDA content, lower relative water content and lower chlorophyll content than BSMV-0 and BSMV-PDS lines after drought stress (Fig. 6d-f).

In addition, we also measured the water loss rate, relative water content and chlorophyll content of wheat leaves from wild-type, TabHLH49-OE and WZY2-RNAi wheat lines. TabHLH49-OE wheat showed the highest relative water and chlorophyll contents and lowest water loss rate compared with the wild-type and WZY2-RNAi wheat lines under drought stress, while the WZY2-RNAi lines showed the lowest relative water and chlorophyll contents and the highest water loss rate (Fig. 7a-c). These results indicated that TabHLH49 positively regulated dehydrin WZY2 gene expression to improve drought resistance in wheat.

\section{Discussion}

TabHLH49 encodes stress-responsive bHLH transcription factor and positively regulates wheat drought tolerance Transcription factors play an important function in regulating the drought response in plants. In Arabidopsis, many bHLH transcription factors are involved in the drought-induced stress response, such as AtbHLH68 [14], AtbHLHOO6 [15], AtbHLH122 [16], AtbHLH17 [17] and AtbHLH92 [18]. However, only a few of bHLHs have been reported in wheat, such as TabHLH1 [19] and TabHLH39 [20], and the regulatory mechanism and biological functions of many other TabHLH proteins remain unclear. Here, we obtained a novel wheat bHLH transcription factor, TabHLH49, through the yeast onehybrid $(\mathrm{Y} 1 \mathrm{H})$ assay with the dehydrin WZY2 promoter as bait. Phylogenetic analysis showed that TabHLH49 was highly homologous to AtbHLH049 and was a Myctype bHLH transcription factor (Fig. 1b). TabHLH49 was obviously induced by drought stress (Fig. 3b), and it enhanced the tolerance to drought stress (Fig. 5c). Compared with the BSMV-0 lines, the BSMV-TabHLH49 lines displayed reduced tolerance to drought stress (Fig. 6a). These results demonstrated that TabHLH49 encodes a novel bHLH transcription factor that positively regulates drought tolerance in wheat.

\section{TabHLH49 positively regulates dehydrin WZY2 gene expression}

Transcription factors regulate the expression of downstream genes by binding cis-acting elements of the promoter. In our previous study, we identified G-box elements in the promoter of WZY2 that bind the bHLH transcription factor [21]. EMSA and $\mathrm{Y} 1 \mathrm{H}$ assays illustrated that TabHLH49 protein can bind and interact with the promoter of WZY2 (Fig. 4). The real-time PCR results indicated that the expression pattern of $W Z Y 2$ was similar to TabHLH49 but lagged behind TabHLH49 (Fig. 3b). Thus, TabHLH49 is a potential positive regulator of WZY2 expression. A dual-luciferase assay also confirmed this result (Fig. 5b). In transient expression analysis, the WZY2 expression level was significantly increased under drought stress in TabHLH49-OE wheat lines compared to CK (Fig. 5e). Similarly, the WZY2 expression level was significantly reduced in BSMVTabHLH49 lines (Fig. 6c). In addition, TabHLH49 protein showed no transcriptional activity in yeast when the C-terminus (amino acids 323-362) was deleted (Fig. 2c). These results indicate that TabHLH49 positively regulates dehydrin $W Z Y 2$ gene expression. 


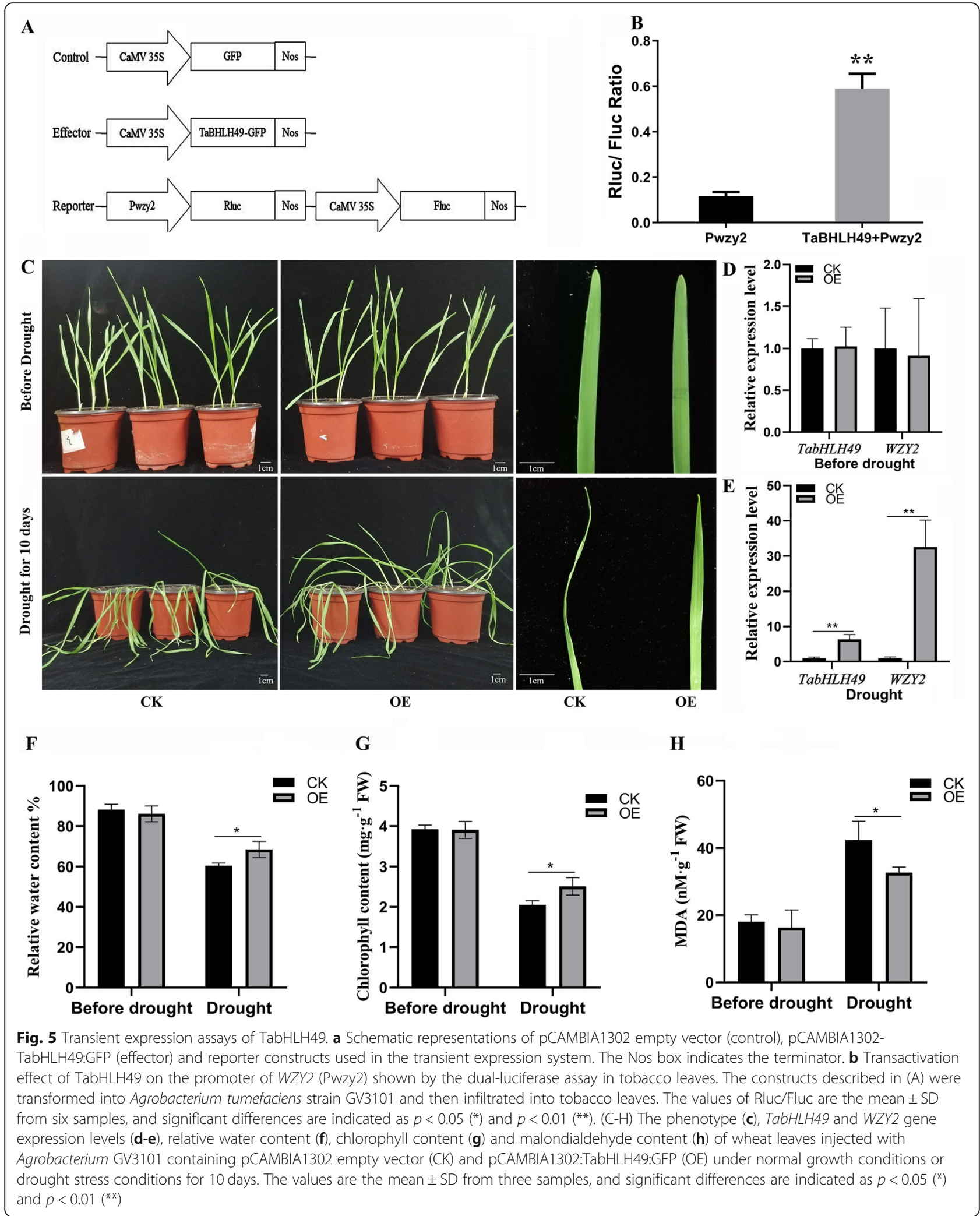




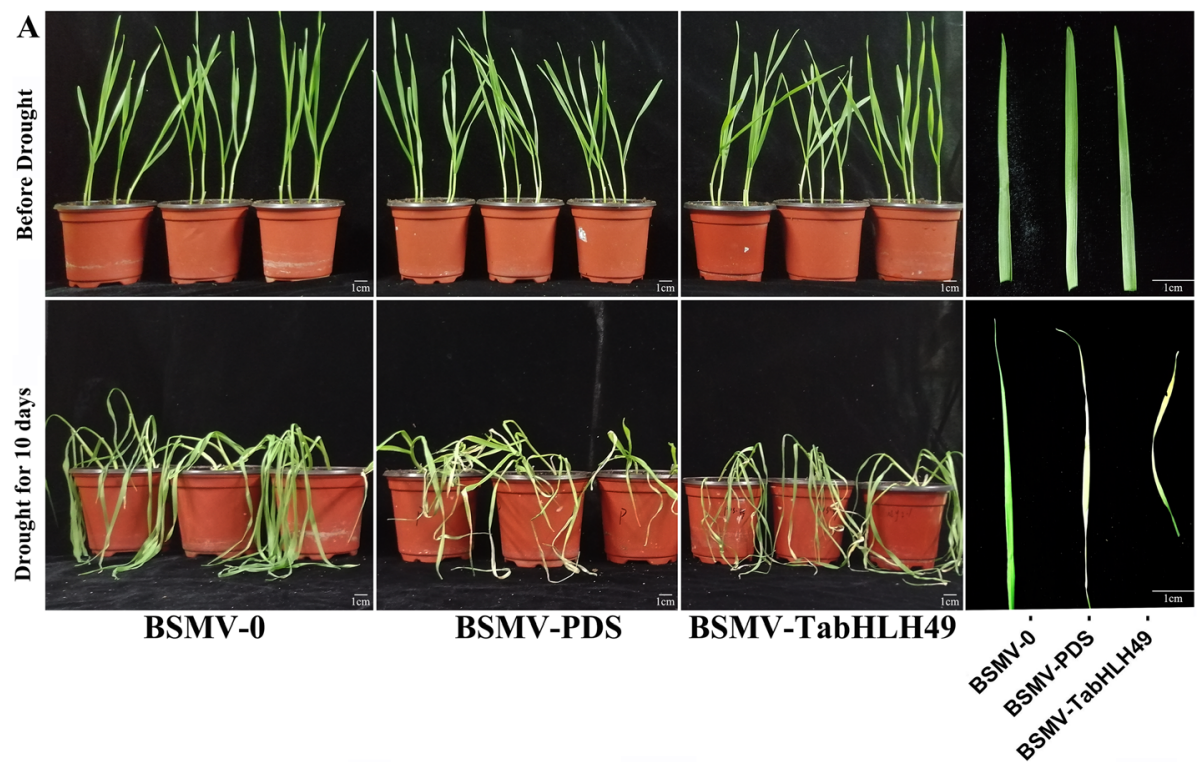

B

C

D
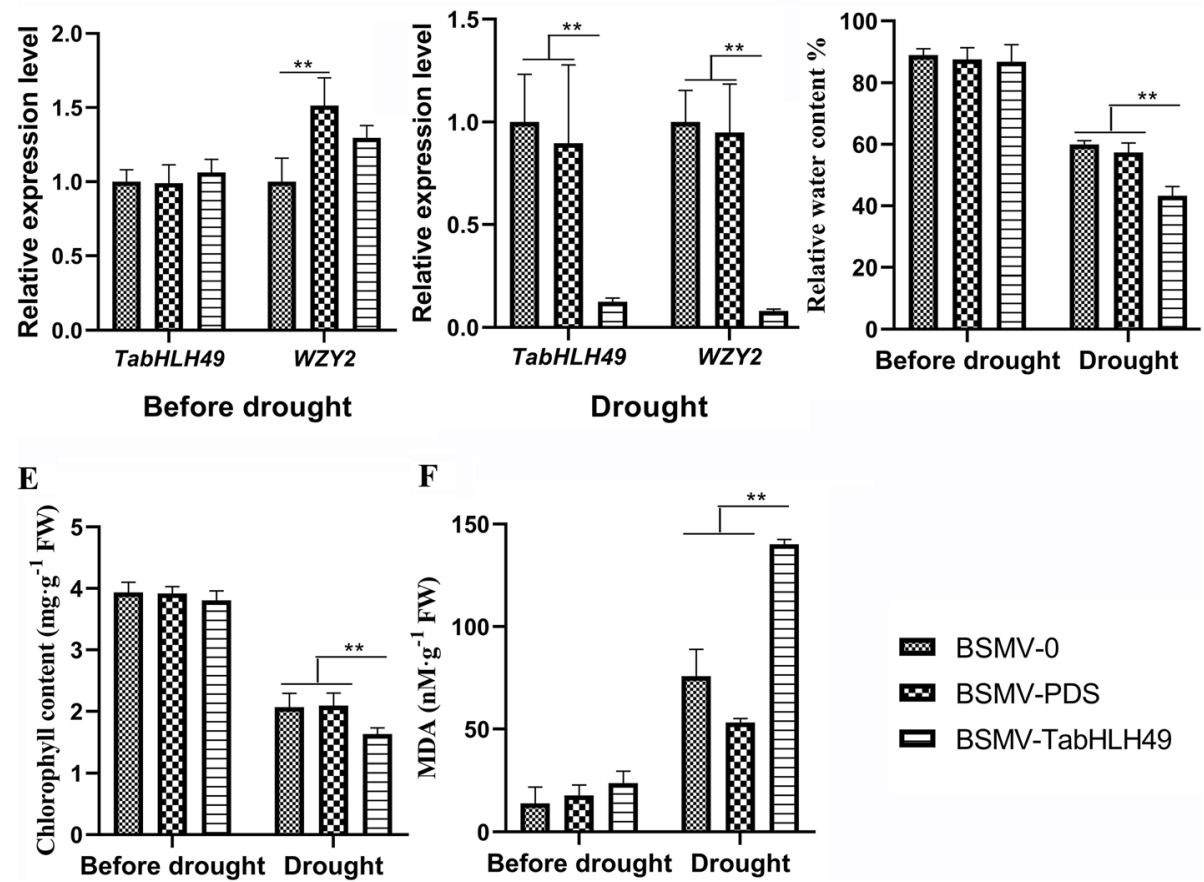

F

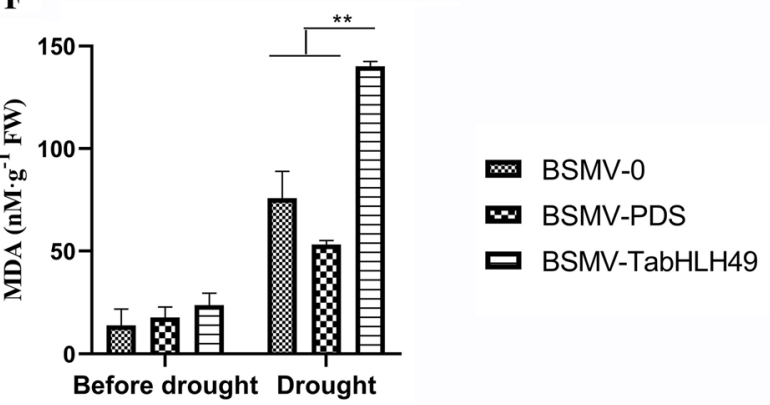

Fig. 6 Virus-induced gene silencing (VIGS) of TabHLH49 in wheat leaves. The phenotype (a), TabHLH49 and WZY2 gene expression levels (b-c), relative water content $(\mathbf{d})$, chlorophyll content $(\mathbf{e})$ and malondialdehyde content (f) of wheat leaves treated with BSMV-0 (empty vector), BSMVPDS and BSMV-TabHLH49 under normal growth conditions or drought stress conditions for 10 days. The values are the mean \pm SD from three samples, and significant differences are indicated as $p<0.05\left(^{*}\right)$ and $p<0.01(* *)$

Taken together, the results presented in this study provide evidence that TabHLH49 positively regulates dehydrin $W Z Y 2$ gene expression to improve the drought resistance of wheat. We further need to develop stably overexpressed TabHLH49 to identify whether this transcription factor participates in stress, which will facilitate investigating the explicit role of TabHLH49 in the dehydrin signaling pathway.

\section{Conclusions}

This study demonstrates that the transcription factor TabHLH49 positively regulates the expression level of 


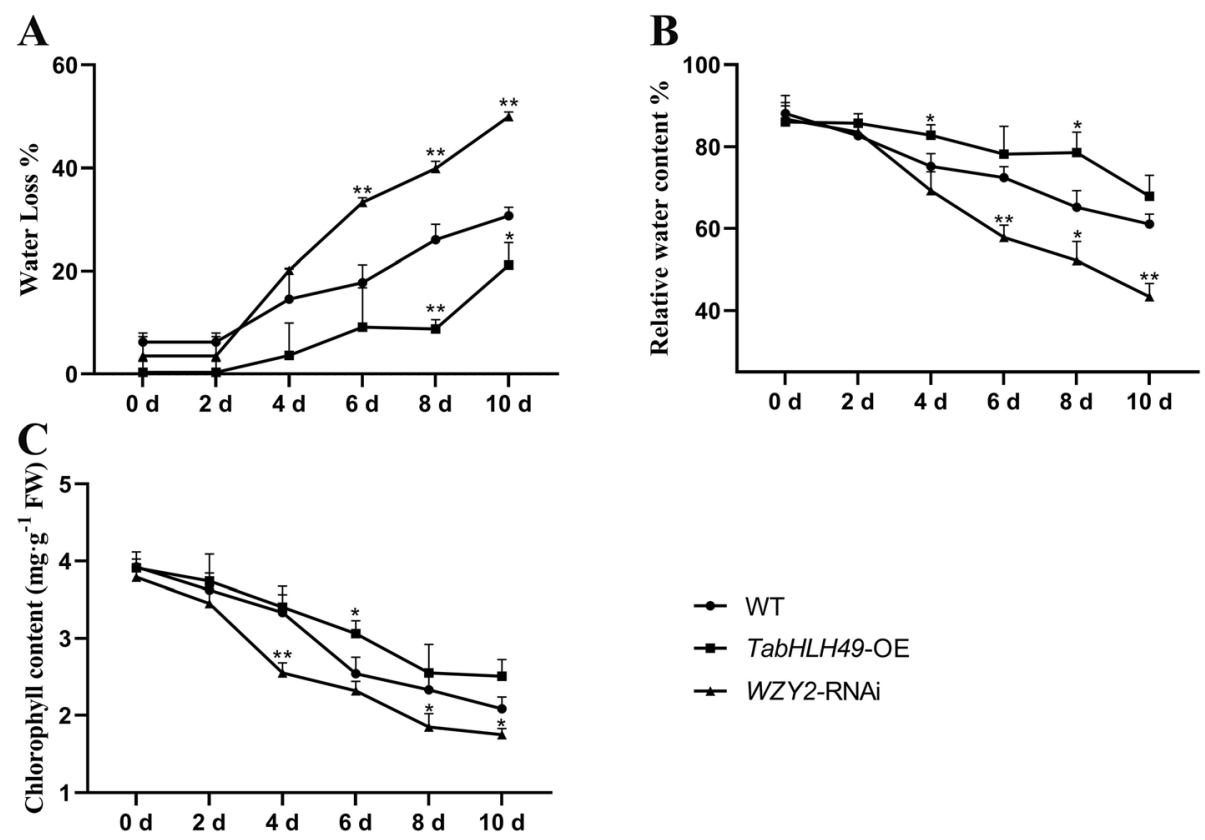

Fig. 7 Determination of water loss rate, relative water content and chlorophyll content in TabHLH49-OE and WZY2-RNAi wheat lines. Water loss rate (a), relative water content (RWC\%) (b) and chlorophyll content (c) of wheat leaves injected with Agrobacterium GV3101 containing pCAMBIA1302 empty vector (CK), pCAMBIA1302:TabHLH49:GFP (TabHLH49-OE) or WZY2-RNAi wheat lines. The values are the mean \pm SD from three samples, and significant differences are indicated as $p<0.05\left(^{*}\right)$ and $p<0.01\left(^{* *}\right)$

the WZY2 gene via binding to its promoter, thereby enhancing the drought resistance in wheat. Our research provides a better understanding of the regulatory mechanism of the WZY2 genes in response to drought stress and offers potential strategies for further crop breeding.

\section{Methods}

Yeast one-hybrid screening of T.aestivum cDNA libraries The $619 \mathrm{bp}$ WZY2 promoter (Pwzy2) was cloned into the pAbAi vector, and the recombinant plasmids were transformed into Y1H Gold yeast cells as bait [10]. Twoweek-old wheat seedlings exposed to either $12 \mathrm{~h}$ high PEG $6000(20 \%)$ or cold $\left(4{ }^{\circ} \mathrm{C}\right)$ treatments were used to construct the cDNA library. The extraction of mRNA was performed with the MiniBEST Plant RNA Extraction kit (Taraka). First-strand cDNA was synthesized by the SMART cDNA Library Construction Kit (Taraka). All fragments were linked to the vector pGADT7-AD and transformed into yeast bait strain Y1H Gold (Pwzy2) following the Yeast Protocols Handbook (Clontech). The transformed stains were cultured on SD/-Leu/AbA ${ }^{200}$ medium. Yeast plasmid was extracted and amplified with the T7 and 3'AD sequencing primers. The amplicons were transformed into Escherichia coli and sequenced.

\section{Bioinformatics analysis of TabHLH49}

The ORF of TabHLH49 was identified in the wheat genomic database of the Ensembl servers (http://plants.
ensembl.org/Triticum_aestivum/Info/Index). The physiochemical characteristics were predicted by the ProtParam tool [22]. The basic helix-loop-helix conserved domain and the subcellular localization were found by the NCBI Conserved Domain database (https://www.ncbi.nlm.nih. gov/cdd) and ProtComp (http://linux1.softberry.com/), respectively. The Swiss-Model tool (https://swissmodel. expasy.org/) was used to predict the three-dimensional structure of the TabHLH49 protein. The phylogenetic tree was constructed by the neighbor-joining method with 1000 bootstrap replicates in MEGA 7.0 software [23]. The amino acid sequences of the wbHLH transcription factors were obtained from a previous report [24].

\section{Subcellular localization of TabHLH49}

To study the subcellular localization of TabHLH49 protein, the Agrobacterium GV3101 carrying the recombinant plasmid pCAMBIA1302:TabHLH49:GFP and pCAMBIA1302 empty vector (control) were injected into Nicotiana benthamiana leaves. The fluorescence signal was observed in tobacco epidermal cells by fluorescence microscopy after culturing in the dark for $36 \mathrm{~h}$ at $28^{\circ} \mathrm{C}$.

\section{Analysis of TabHLH49 transactivation}

A transcript activation activity assay was carried out as previously reported [8]. The full-length and truncated fragments of TabHLH49 were fused with GAL4 DNA- 
binding domain of the pGBKT7 plasmid and transformed into yeast $\mathrm{Y} 2 \mathrm{H}$ Gold cells, and then grown on SD-Trp/X- $\alpha$-gal/AbA plates to examine their growth and $\alpha$-galactosidase activity.

\section{Plant materials and growth conditions}

Wheat seeds of "ZhengYin 1\#" were used throughout this study. Growth condition of wheat plants and 20\% PEG6000 treatment were the same as that described in our previous study [25].

\section{RNA isolation and real-time PCR analysis}

The protocol of RNA isolation and real-time PCR were described as our previous study [25]. All real-time PCR reactions were performed in triplicate to ensure reproducibility of the results.

\section{Expression of fusion TabHLH49 protein and purification} TabHLH49 was cloned into pET-28a vector, and recombinant plasmid was transformed into competent BL21 (DE3) cells. The expression of recombinant protein was induced with $1 \mathrm{mM}$ isopropyl $\beta$-D-1-thiogalactopyranoside (IPTG) for $6 \mathrm{~h}$ after the recombinant $E$. coli density reached an $\mathrm{OD}_{600}$ of 0.6 in $\mathrm{LB}$ liquid medium containing with $50 \mu \mathrm{g} / \mathrm{mL}$ kanamycin at $37^{\circ} \mathrm{C}$. The cells were harvested by centrifugation and were resuspended in phosphate-buffered saline (PBS), followed by ultrasonic cell disruption for $30 \mathrm{~min}$. The TabHLH49 protein was purified using ProteinIso ${ }^{\mathrm{ma}}$ Ni-NTA resin (TransGen) and detected by SDS-PAGE.

\section{Electrophoretic mobility shift assay}

EMSA was performed with the LightShift ${ }^{\circ} \mathrm{Chemilumin-}^{-}$ escent EMSA Kit (Thermo Fisher Scientific) following the manufacturer's protocol. The $619 \mathrm{bp}$ region of the WZY2 promoter sequences were amplified using genespecific primers (Table S1) and then purified and quantified. The WZY2 promoter was reacted with the purified TabHLH49 protein at room temperature for $30 \mathrm{~min}$. The complexes were then separated by native $6 \%$ polyacrylamide gel electrophoresis.

\section{Dual-luciferase assay}

To investigate the transactivation role of TabHLH49 on the target WZY2 promoter, a dual-luciferase assay was conducted according to previously reported [26]. The promoter of $W Z Y 2$ was cloned into the dual-luciferase report vector pC0390-RUC and transformed into Agrobacterium GV3101 and then injected together with either pCAMBIA1302:TabHLH49:GFP (effector) or pCAMBIA1302 empty vector (control) into tobacco leaves. After incubation for $3 \mathrm{~d}$, the leaves were ground in liquid nitrogen and then mixed with passive lysis buffer at room temperature for $30 \mathrm{~min}$. After centrifugation, the supernatant was collected and assessed for luciferase activity (RLuc/Fluc) using the Dual-Luciferase ${ }^{\circ}$ Reporter Assay System (Promega).

\section{Transient expression analysis of TabHLH49}

The Agrobacteria GV3101 transformed with the pCAMBIA1302:TabHLH49:GFP and pCAMBIA1302 empty vector (control) were injected into wheat leaves for transient gene expression assays, as previously described [12, 13]. Fresh Agrobacteria were grown overnight in yeast extract broth (YEB) medium with rifampicin, carbenicillin, and spectinomycin. After centrifugation, the bacterial pellets were resuspended in an infiltration buffer containing $10 \mathrm{mM}$ MES, $10 \mathrm{mM} \mathrm{MgCl}_{2}$, and $400 \mu \mathrm{M}$ acetosyringone at an optical density of $\mathrm{OD}_{600}=2.0$. For wheat infiltration, the fully expanded secondary leaf of wheat seedlings was infiltrated using a $1 \mathrm{~mL}$ syringe without a needle. Plant phenotypes were examined between 5 and $14 \mathrm{~d}$ after infiltration.

\section{BSMV-mediated TabHLH49 gene silencing}

In vitro transcription of viral RNA and plant inoculations were performed as previously described [27]. Four plants of wheat were infected with BSMV-PDS (positive control) or BSMV-TabHLH49 construct, with inoculation with BSMV:0 (negative control) was used as a control in each experiment. This experiment was repeated three times, and the data were averaged.

\section{Determination of malondialdehyde (MDA), water loss, relative water content and chlorophyll content}

Determination of the malondialdehyde (MDA) content, the water loss rate, the relative water content and the chlorophyll content of wheat leaves after drought stress was performed as previously described [11, 28, 29].

\section{Supplementary information}

Supplementary information accompanies this paper at https://doi.org/10 1186/s12870-020-02474-5.

Additional file 1: Table S1. Specific primers used in the study.

Additional file 2: Figure S1. Raw images of Fig. 4a.

Additional file 3: Figure S2. Raw images of Fig. 4b.

\section{Abbreviations}

bHLH: Basic helix-loop-helix transcription factor; Pwzy2: Promoter of the dehydrin WZY2 gene; assay; Rluc: Renilla luciferase; Fluc: Firefly luciferase; AbA: Aureobasidin A; Y1H: Yeast one-hybrid; IPTG: Isopropyl $\beta$-D-1-thiogalactopyranoside; MDA: Malondialdehyde; VIGS: Virus-induced gene silencing

\section{Acknowledgements}

We acknowledge Dr. Tianyong Zhao from Northwest A\&F University for providing a dual-luciferase reporting vector and Dr. Zhensheng Kang from Northwest A\&F University for providing the BSMV-VIGS vector. We also acknowledge the National Natural Science Foundation of China (No. 31671608). 


\section{Authors' contributions}

Designed the experiment: HL and LZ. Performed the experiments and analyzed data: $\mathrm{HL}$ and $Y Y$. Contributed reagents/materials/analysis tools: $L Z$. Wrote the manuscript: HL, YY, XW and DL. All authors read and approved the manuscript.

\section{Funding}

Our research was funded by the National Natural Science Foundation of China (Grant No. 31671608). The funding agencies had no role in the experimental design, data collection and analysis or preparation of the manuscript.

\section{Availability of data and materials}

The datasets generated and analysed during the current study are available in the UniProt database with the link of https://www.uniprot.org/, under the accession number BOLXL4 and A0A3B6RC19, respectively. The sequences of WZY2 promoter can be found in the additional files of our previous study with the link of https://doi.org/10.1080/15592324.2019.1678370.

\section{Ethics approval and consent to participate}

Not applicable.

\section{Consent for publication}

Not applicable.

\section{Competing interests}

The authors declare that they have no competing interests.

\section{Author details}

${ }^{1}$ College of Life Science/State Key Laboratory of Crop Stress Biology for Arid Areas, Northwest A\&F University, Yangling 712100, China. ${ }^{2}$ College of Nursing, Weinan Vocational\&Technical College, Weinan 714000, China. ${ }^{3}$ School of Agriculture, Yunnan University, Kunming 650000, China. ${ }^{4}$ Institute of Evolution \& Marine Biodiversity, Ocean University of China, Qingdao 266000, China.

\section{Received: 9 October 2019 Accepted: 27 May 2020}

\section{Published online: 05 June 2020}

\section{References}

1. Battaglia M, Olvera-Carrillo Y, Garciarrubio A, Campos F, Covarrubias AA The enigmatic LEA proteins and other hydrophilins. Plant Physiol. 2008; 148(1):6-24.

2. Garay-Arroyo A, Colmenero-Flores JM, Garciarrubio A, Covarrubias AA. Highly hydrophilic proteins in prokaryotes and eukaryotes are common during conditions of water deficit. J Biol Chem. 2000;275(8):5668-74.

3. Yu Z, Wang $X$, Zhang L. Structural and Functional Dynamics of Dehydrins: A Plant Protector Protein under Abiotic Stress. Int J Mol Sci. 2018:19(11):3420.

4. Atchley WR, Terhalle W, Dress A. Positional dependence, cliques, and predictive motifs in the bHLH protein domain. J Mol Evol. 1999;48(5): 501-16.

5. Nair SK, Burley SK. Recognizing DNA in the library. Nature. 2000;404(6779): 715 717-718.

6. Zhang W, Sun Y, Timofejeva L, Chen C, Grossniklaus U, Ma H. Regulation of Arabidopsis tapetum development and function by DYSFUNCTIONAL TAPETUM1 (DYT1) encoding a putative bHLH transcription factor. Development. 2006;133(16):3085-95.

7. Heisler M, Atkinson A, Bylstra YH, Walsh R, Smyth DR. SPATULA, a gene that controls development of carpel margin tissues in Arabidopsis, encodes a bHLH protein. DEVELOPMENT. 2001;128(7):1089-98.

8. Liu Y, Ji X, Nie X, Qu M, Zheng L, Tan Z, Zhao H, Huo L, Liu S, Zhang B, et al. Arabidopsis AtbHLH112 regulates the expression of genes involved in abiotic stress tolerance by binding to their E-box and GCG-box motifs. New Phytol. 2015;207(3):692-709.

9. Ji X, Nie X, Liu Y, Zheng L, Zhao H, Zhang B, Huo L, Wang Y. A bHLH gene from Tamarix hispida improves abiotic stress tolerance by enhancing osmotic potential and decreasing reactive oxygen species accumulation. Tree Physiol. 2016;36(2):193-207.

10. Liu H, Liu H, Yang Y, Zhang L. Identification of upstream transcription factors and an interacting PP2C protein of dehydrin WZY2 gene in wheat. Plant Signal Behav. 2019;14(12):1678370.
11. Yu Z, Wang $X, M u X$, Zhang L. RNAi mediated silencing of dehydrin gene WZY2 confers osmotic stress intolerance in transgenic wheat. Funct Plant Biol. 2019;46:877-84.

12. Lu X, Kracher B, Saur IML, Bauer S, Ellwood SR, Wise R, Yaeno T, Maekawa T, Schulze-Lefert $P$. Allelic barley MLA immune receptors recognize sequenceunrelated avirulence effectors of the powdery mildew pathogen. Proc Natl Acad Sci. 2016;113(42):E6486-95.

13. Gao J, Bi W, Li H, Wu J, Yu X, Liu D, Wang X. WRKY transcription factors associated with NPR1-mediated acquired resistance in barley are potential resources to improve wheat resistance to Puccinia triticina. Front Plant Sci. 2018;9:1486.

14. Le Hir R, Castelain M, Chakraborti D, Moritz T, Dinant S, Bellini C. AtbHLH68 transcription factor contributes to the regulation of ABA homeostasis and drought stress tolerance in Arabidopsis thaliana. Physiol Plantarum. 2017; 160(3):312-27.

15. Abe H, Urao T, Ito T, Seki M, Shinozaki K, Yamaguchi-Shinozaki K. Arabidopsis AtMYC2 (bHLH) and AtMYB2 (MYB) function as transcriptional activators in abscisic acid signaling. Plant Cell. 2003;15(1):63-78.

16. Liu W, Tai H, Li S, Gao W, Zhao M, Xie C, Li WX. bHLH122 is important for drought and osmotic stress resistance in Arabidopsis and in the repression of ABA catabolism. New Phytol. 2014;201(4):1192-204.

17. Babitha KC, Ramu SV, Pruthvi V, Mahesh P, Nataraja KN, Udayakumar M. Coexpression of AtbHLH17 and AtWRKY28 confers resistance to abiotic stress in Arabidopsis. Transgenic Res. 2013;22(2):327-41.

18. Jiang Y, Yang B, Deyholos MK. Functional characterization of the Arabidopsis bHLH92 transcription factor in abiotic stress. Mol Gen Genomics. 2009;282(5):503-16.

19. Yang T, Yao S, Hao L, Zhao Y, Lu W, Xiao K. Wheat bHLH-type transcription factor gene TabHLH1 is crucial in mediating osmotic stresses tolerance through modulating largely the ABA-associated pathway. Plant Cell Rep. 2016;35(11):2309-23.

20. Zhai Y, Zhang L, Xia C, Fu S, Zhao G, Jia J, Kong X. The wheat transcription factor, TabHLH39, improves tolerance to multiple abiotic stressors in transgenic plants. Biochem Biophys Res Commun. 2016;473(4):1321-7.

21. Zhu W, Zhang L, Lv H, Zhang H, Zhang D, Wang X, Chen J. The dehydrin wzy2 promoter from wheat defines its contribution to stress tolerance. Funct Integr Genomic. 2014;14(1):111-25.

22. Garg VK, Avashthi H, Tiwari A, Jain PA, Ramkete PW, Kayastha AM, Singh VK. MFPPI - multi FASTA ProtParam Interface. Bioinformation. 2016;12(2):74-7.

23. Kumar S, Stecher G, Tamura K. MEGA7: molecular evolutionary genetics analysis version 7.0 for bigger datasets. Mol Biol Evol. 2016;33(7):1870-4.

24. Guo X, Wang J. Global identification, structural analysis and expression characterization of bHLH transcription factors in wheat. BMC Plant Biol. 2017;17(1):90

25. Liu H, Xing M, Yang W, Mu X, Wang X, Lu F, Wang Y, Zhang L. Genomewide identification of and functional insights into the late embryogenesis abundant (LEA) gene family in bread wheat (Triticum aestivum). Sci Rep UK. 2019;9(1):13375

26. Chen K, Du L, Liu H, Liu Y. A novel R2R3-MYB from grape hyacinth, MaMybA, which is different from MaAN2, confers intense and magenta anthocyanin pigmentation in tobacco. BMC Plant Biol. 2019;19(1):390.

27. Holzberg S, Brosio P, Gross C, Pogue GP. Barley stripe mosaic virus-induced gene silencing in a monocot plant. Plant J. 2002;30(3):315-27.

28. Kang H, Zhang M, Zhou S, Guo Q, Chen F, Wu J, Wang W. Overexpression of wheat ubiquitin gene, ta-Ub2, improves abiotic stress tolerance of Brachypodium distachyon. Plant Sci. 2016;248:102-15.

29. Zhang G, Zhang M, Zhao Z, Ren Y, Li Q, Wang W. Wheat TaPUB1 modulates plant drought stress resistance by improving antioxidant capability. Sci Rep UK. 2017;7(1):7549.

\section{Publisher's Note}

Springer Nature remains neutral with regard to jurisdictional claims in published maps and institutional affiliations. 